\title{
Permutation-symmetric critical phases in disordered non-Abelian anyonic chains
}

\author{
L. Fidkowski and H.-H. Lin \\ Institute for Quantum Information, California Institute of Technology, MC 114-36, Pasadena, California 91125, USA \\ P. Titum \\ Indian Institute of Technology Kanpur, Kanpur, Uttar Pradesh 208016, India \\ G. Refael \\ Department of Physics, Institute for Quantum Information, California Institute of Technology, MC 114-36, Pasadena, California 91125, \\ USA
}

(Received 22 January 2009; published 27 April 2009)

\begin{abstract}
Topological phases supporting non-Abelian anyonic excitations have been proposed as candidates for topological quantum computation. In this paper, we study disordered non-Abelian anyonic chains based on the quantum groups $\mathrm{SU}(2)_{k}$, a hierarchy that includes the $\nu=5 / 2$ fractional quantum Hall state and the proposed $\nu=12 / 5$ Fibonacci state, among others. We find that for odd $k$ these anyonic chains realize infinite-randomness critical phases in the same universality class as the $S_{k}$ permutation symmetric multicritical points of Damle and Huse [Phys. Rev. Lett. 89, 277203 (2002)]. Indeed, we show that the pertinent subspace of these anyonic chains actually sits inside the $\mathbb{Z}_{k} \subset S_{k}$ symmetric sector of the Damle-Huse model, and this $\mathbb{Z}_{k}$ symmetry stabilizes the phase.
\end{abstract}

DOI: 10.1103/PhysRevB.79.155120

PACS number(s): 75.10.Pq

\section{INTRODUCTION}

One of the major advances in the understanding of strongly correlated quantum systems has been the exploration of topological phases of matter. Originating with the discovery of the $\nu=1 / 3$ fractional quantum Hall effect, topological phases have received much renewed interest with their recent proposed application to quantum computation. ${ }^{1}$ Under this proposal, quantum computation is carried out by the braiding of the non-Abelian quasiparticle excitations of the topological phase. The topologically protected degenerate space of ground states of the non-Abelions serves as the memory, and the braiding induces unitary transformations within this Hilbert space. ${ }^{2}$ The remarkable feature of this scheme is that the dimension of this space for $N$ anyons grows as $d^{N}$, with the quantum dimension $d$ in general noninteger. This should be contrasted, for example, with $N$ spin$1 / 2$ quasiparticles, whose Hilbert space has $2^{N}$ states. The nonintegral nature of $d$ reflects a unique nonlocality of the anyon Hilbert space and makes decoherence-free quantum computation possible: no local perturbations can give rise to decoherence.

The potential applications of topological phases with nonAbelian anyons, and perhaps even more so their remarkable properties, warrant an analysis of interacting many-anyon systems. Possibly the most natural starting point is a onedimensional anyonic chain, analogous to regular spin chains such as the SU(2) Heisenberg model. Though such anyonic chains do not have truly local degrees of freedom, they can be written as local systems with local constraints. It was found that even rather simple to construct translationally invariant chains, based on $\mathrm{SU}(2)_{3}$ anyons, have an intricate structure, with the low-energy degrees of freedom organized into the gapless spectra of the $c=4 / 5$ and $c=7 / 10$ conformal field theories. ${ }^{3}$ These chains interact via nearest-neighbor couplings and are exactly solvable, though more generalized models exhibit an even richer set of critical and gapped phases. ${ }^{4}$

Whereas translationally invariant chains are the requisite first step, a more likely physical realization of a non-Abelian chain, for example in a quantum Hall state, will have a strong degree of quenched disorder. Such chains are the focus of this paper. Even with garden-variety spins, the addition of disorder dramatically affects the physics, with the low-energy behavior controlled by so-called infiniterandomness fixed points. ${ }^{5,6} \mathrm{~A}$ prototypical example is the random singlet phase describing the state of a disordered spin-1/2 Heisenberg chain. Here spins pair up and form singlets in a random fashion, with most connecting near neighbors but some being very long ranged. This unique structure of the ground state leads to some rather unexpected or unusual properties, including algebraically decaying average correlations and energy-length scaling $|\ln E| \sim L^{\psi}$ as opposed to the usual $1 / E \sim L^{z}$ characteristic of pure systems. Furthermore, disordered spin- $S$ chains with $S>1 / 2$ were shown to exhibit infinite-randomness critical fixed points with the universal exponent $\psi=1 /(2 S)$, and with the spin state described as a competition between $2 S+1$ domains, as we describe below. These fixed points were dubbed the $S_{n}$ permutation symmetric points, with $n=2 S+1$ the number of competing domains. ${ }^{7-10}$

Because of the unique structure of their Hilbert spaces, disordered anyonic chains are particularly amenable to treatment via strong randomness renormalization-group (RG) methods and indeed have been shown to exhibit infiniterandomness fixed points. ${ }^{11,12}$ They are thus an especially fertile ground for trying to discover and classify new universality classes of strongly random behavior. Indeed, even though no new universality classes were found, Ref. 12 found a rich phase diagram for the $\mathrm{SU}(2)_{3}$, or Fibonacci anyonic chain, with a random singlet fixed point that can be destabilized by the addition of couplings favoring fusion into a nontrivial 
topological charge, and a resulting flow to a more intricate $S_{3}$ permutation symmetric fixed point. It is notable that this $S_{3}$ symmetric point is actually a critical phase, with no relevant perturbations - this contrasts with the domain realization of the $S_{n}$ symmetric fixed point, which has $n-1$ relevant perturbations. $^{7}$

The non-Abelian models that are the subject of this paper are based on $\mathrm{SU}(2)_{k}$ anyons with $k$ odd. The level $k$ signifies a truncation of all representations with spin $S>k / 2$ - the anyons of $\mathrm{SU}(2)_{k}$ simply correspond to the first $k+1$ irreducible representations of $\mathrm{SU}(2)$. This fact potentially suggests a subtle analogy between the $\mathrm{SU}(2)_{k}$ systems and spins $S$ truncated at $k / 2$. In particular, it raises the possibility of a relation between the $S_{k}$ permutation symmetric fixed points of regular spin- $(k-1) / 2$ chains, and the infinite-randomness phases arising in $\mathrm{SU}(2)_{k}$ anyons.

In this paper we show that, indeed, for all odd $k, \mathrm{SU}(2)_{k}$ anyonic chains realize $S_{k}$ symmetric infinite-randomness critical phases. Crucial to our analysis is expressing the twoanyon interaction terms in a novel basis, one which behaves better than the standard projector basis with respect to the $\mathrm{RG}$ decimations. With this insight, we are able to solve the model, and in fact construct an explicit equivalence between it and the $\mathbb{D}_{k} \subset S_{k}$ symmetric sector of the domain model of Damle and Huse, ${ }^{7}$ where the order $2 k$ dihedral group $\mathrm{D}_{k}$ in particular contains $\mathbb{Z}_{k}$. The $\mathbb{Z}_{k}$ symmetry is what stabilizes the phase, eliminating the $k-1$ relevant perturbations of the domain model. This phenomenon of a $k$ multicritical point being stabilized by additional symmetry in the $\mathrm{SU}(2)_{k}$ model is tantalizingly close to that discovered in Ref. 13 for the uniform $\mathrm{SU}(2)_{k}$ chain. There, stability of an a priori $k$ multicritical point is guaranteed by an extra "topological" symmetry in the quantum system. It is conceivable that these two phenomena are closely related.

The rest of this paper is structured as follows. In Sec. II we briefly review necessary background on the strong randomness renormalization-group procedure, as well as set up the construction of the anyon chain Hilbert space and Hamiltonian. In Sec. III we analyze the disordered anyon chain, introducing the novel basis for the interaction terms. We write down and solve the flow equations, finding a fixed point of the RG. In Sec. IV we construct an explicit equivalence between the disordered anyon chain and the $\mathrm{D}_{k}$ symmetric sector of Damle-Huse domain wall model, one that relates their respective fixed points. We conclude in Sec. V and relegate some technical derivations to the Appendix.

\section{BACKGROUND AND SETUP}

\section{A. Real-space renormalization group}

To find the ground state of disordered spin chains, Ma and Dasgupta introduced the strong disorder real-space renormalization-group method. ${ }^{14,15}$ The random spin-1/2 Heisenberg model provides the simplest example amenable to such a treatment. The model is given by

$$
H=\sum_{i} J_{i, i+1} \mathbf{S}_{i} \cdot \mathbf{S}_{i+1}
$$

where the couplings $J_{i, i+1}>0$ are positive and randomly distributed. Note that, as far as the Hilbert space is concerned, we have for two neighboring sites

$$
\frac{1}{2} \otimes \frac{1}{2}=0 \oplus 1
$$

and the interaction $\mathbf{S}_{i} \cdot \mathbf{S}_{i+1}$ simply gives an energy splitting between the singlet and triplet. The procedure now is to pick the largest $J_{i, i+1}$, which effectively decimates the excited triplet and leaves the ground state in a singlet, and do perturbation theory around that state. Quantum fluctuations then induce an effective coupling according to the so-called MaDasgupta rule: ${ }^{14,15}$

$$
J_{i-1, i+2}=\frac{J_{i-1, i} J_{i+1, i+2}}{2 J_{i, i+1}} .
$$

So sites $i$ and $i+1$ are decimated and replaced with an effective interaction between $i-1$ and $i+2$. Iteration of this procedure produces singlet bonds on all length scales. This is the random singlet ground state.

A quantitative description is obtained by tracking the $R G$ flow of the coupling distribution. It is useful to employ logarithmic couplings: ${ }^{5}$

$$
\beta_{i, i+1}=\ln \frac{\Omega}{J_{i, i+1}},
$$

where $\Omega=\max _{i} J_{i, i+1}$. In these variables the Ma-Dasgupta rule (3) reads

$$
\beta_{i-1, i+2}=\beta_{i-1, i}+\beta_{i+1, i+2}
$$

(up to an additive constant of $\ln 2$, which can be safely neglected). As the couplings get decimated $\Omega$ decreases. It is convenient to define the RG flow parameter as

$$
\Gamma=\ln \frac{\Omega_{0}}{\Omega},
$$

where $\Omega_{0}$ is the maximal coupling of the bare Hamiltonian. Let $P_{\Gamma}(\beta)$ be the distribution of couplings. We can derive a flow equation for $P_{\Gamma}(\beta)$ by decimating the couplings in the infinitesimal interval $\beta=[0, d \Gamma]$ and seeing how their probabilistic weight is redistributed. We obtain

$$
\begin{aligned}
\frac{d}{d \Gamma} P_{\Gamma}(\beta)= & \frac{\partial P_{\Gamma}}{\partial \beta}+P(0) \int_{0}^{\infty} d \beta_{1} \\
& \times \int_{0}^{\infty} d \beta_{2} \delta\left(\beta-\beta_{1}-\beta\right) P_{\Gamma}\left(\beta_{1}\right) P_{\Gamma}\left(\beta_{2}\right) .
\end{aligned}
$$

The first term comes from the overall change of scale, and the second from the Ma-Dasgupta rule. These equations have a solution

$$
P_{\Gamma}(\beta)=\frac{1}{\Gamma} e^{-\beta / \Gamma},
$$

which is an attractive fixed point to essentially all physical initial configurations. This solution permits us to read off features of the random singlet phase; for example one can with a little more work derive the energy-length scaling relation 
a)

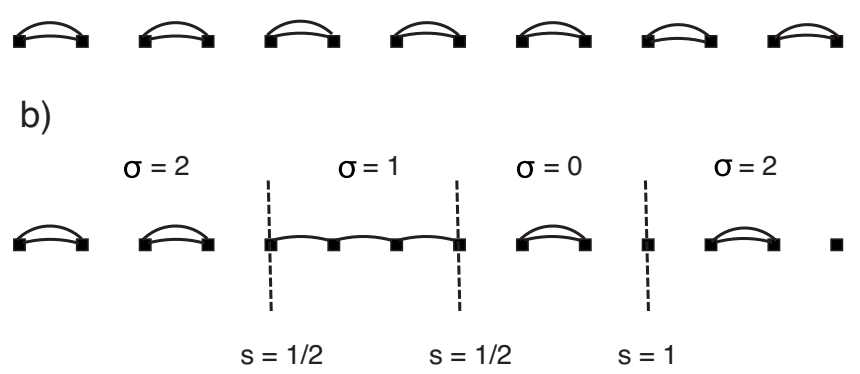

FIG. 1. (a) Valence bond solid of type $\sigma=2$. (b) Sample configuration at the infinite-randomness fixed point. All the domains occur equally. The dashed lines represent domain walls, which contain the unpaired spins that form the low-energy degrees of freedom.

$$
L^{1 / 2} \sim \Gamma=\ln (1 / E),
$$

which thus has the exponent

$$
\psi=1 / 2 \text {. }
$$

The random singlet phase describes the universal lowenergy behavior of several known one-dimensional systems, making it interesting to attempt to classify all such universal low-energy fixed points of strongly random systems in one dimension. Thus far all known universality classes are realized in the Damle-Huse hierarchy of permutation symmetric critical points, ${ }^{7}$ indexed by a positive integer $n$. In the construction of Damle and Huse, the system indexed by $n$ is realized in a spin- $n / 2 \mathrm{SU}(2)$ invariant Heisenberg model, with the sites represented as symmetrized tensor products of spin $1 / 2$ 's. The interaction Hamiltonian is

$$
H=\sum_{i} J_{i} \overrightarrow{\mathbf{S}}_{i} \cdot \overrightarrow{\mathbf{S}}_{i+1}
$$

where the couplings $J_{i}$ contain dimerization $\delta$ and randomness of strength $R$,

$$
J_{i}=J\left[1+\delta(-1)^{i}\right] \exp \left(R \eta_{i}\right) .
$$

Here the $\eta_{i}$ are random variables. Depending on $R$ and $\delta$ this Hamiltonian can realize a plethora of phases, which can be qualitatively understood in terms of valence bond solids (VBS). In this picture, a VBS of type $\sigma \in\{0,1, \ldots, n\}$ is constructed by pairing up $\sigma$ spin-1/2's into singlets over each even bond and $n-\sigma$ spin-1/2's over each odd bond [Fig. 1(a)]. This exhausts the spin degrees of freedom and defines a unique state.

The $S_{n}$ permutation symmetric infinite-randomness fixed point is now realized as a multicritical point in which all of these domains occur equally [Fig. 1(b)]. The domain walls contain unpaired spins: between $\sigma$ and $\sigma^{\prime}$ we have a spin of magnitude $\left|\sigma-\sigma^{\prime}\right| / 2$. These spins interact via effective couplings whose magnitude is highly dependent on the domain that separates them, and whose sign is dictated by a consistency condition. Specifically, for three domains $\sigma_{1}, \sigma_{2}$, and $\sigma_{3}$, the interaction between $\left|\sigma_{1}-\sigma_{2}\right| / 2$ and $\left|\sigma_{2}-\sigma_{3}\right| / 2$ is antiferromagnetic if $\sigma_{1}-\sigma_{2}$ and $\sigma_{3}-\sigma_{2}$ are of the same sign and ferromagnetic otherwise. The fixed point turns out to contain an entirely random distribution of domains, described by a stochastic transfer matrix with all nonzero entries equal to $1 / n$, an energy-length infinite-randomness scaling exponent $\psi=1 / n$, and a logarithmic distribution of the coupling strengths: $P(\beta)=(n / \Gamma) e^{-n \beta / \Gamma}$.

We will see in the remainder of the paper that this multicritical point will be realized (for $n+1$ odd) as a stable phase of the $\mathrm{SU}(2)_{k}$ anyon chain. First, however, we need to review some background on anyonic spin chains.

\section{B. Hilbert space and Hamiltonian of $\mathrm{SU}(2)_{k}$ anyon chains}

A crucial part of our analysis relies on the specific properties of $\mathrm{SU}(2)_{k}$. The nontrivial anyons in this case correspond to the nontrivial representations of $\mathrm{SU}(2)$ at level $k$. There are $k$ of these, labeled by their spin: $0,1 / 2,1, \ldots, k / 2$. The fusion rules are

$$
i \otimes j=\sum_{m=|i-j|}^{\min (i+j, k-i-j)} m,
$$

where $m$ is summing over the integers if $i-j$ is an integer, and over the half integers otherwise. More information is encoded in the so-called $F$ matrix or set of $q-6-j$ symbols of $\mathrm{SU}(2)_{k}$. Given three anyons $j_{1}, j_{2}$, and $j_{3}$, we can either fuse $j_{1}$ and $j_{2}$ first into $j_{12}$ and then with $j_{3}$ into $j$, or we could first fuse $j_{2}$ and $j_{3}$ into $j_{23}$ and then with $j_{1}$ into $j$. Both of these procedures generate a basis for the Hilbert space of ground states of $j_{1}, j_{2}$, and $j_{3}$. The transformation between these two bases is encoded in the $F$-matrix $\left(F_{j_{1} j_{2}}^{j_{3}{ }_{2}}\right)_{j_{12}}^{j_{23}}$. The $F$ matrix of $\mathrm{SU}(2)_{k}$ is written down in the Appendix.

Let us now construct the Hilbert space for the problem. We have a chain $\left\{i_{p}\right\}$ of anyons indexed by an integer position $p$. From now on we will deal only with odd level $k$ and integer values of the "spin"- thus $i_{p} \in\{1, \ldots,(k-1) / 2\}$. We can do this because the integers form a closed fusion subalgebra of $\mathrm{SU}(2)_{k}$. Indeed, there is a $\mathbb{Z}_{2}$ symmetry that relates charge $j$ and $k / 2-j$, and this symmetry will become important when we relate the anyon model to the Damle-Huse model.

There are two equivalent ways to define the Hilbert space. ${ }^{12}$ The simplest construction is to label each "link" between site $p$ and $p+1$ with an integer anyon type $l_{p, p+1}$, subject to the constraint that the fusion rules be obeyed at each site, i.e.,

$$
l_{p, p+1} \in\left\{\left|l_{p-1, p}-i_{p}\right|, \ldots, \min \left(l_{p-1, p}+i_{p}, k-l_{p-1, p}-i_{p}\right)\right\}
$$

See, for example, Fig. 2. The set of all such admissible labelings defines a basis for the Hilbert space, which is simply the space of degenerate ground states of this configuration of anyons.

We can impose the link basis constraints as high energy two-body interactions. The advantage of the link basis is then that it gives us a local way to describe the degrees of freedom in the problem. Indeed, we will see that the Hamiltonian defined below will consist of three-local interactions. In order to define the Hamiltonian, however, it is useful to first 


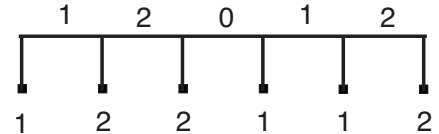

FIG. 2. Sample link basis vector for an $\mathrm{SU}(2)_{5}$ chain. The anyons are represented by the black boxes at the bottom and labeled with their topological charge, 1 or 2 . The links, or bonds, between the anyons are labeled with 0,1 , or 2 , in such a way that the fusion rules at the trivalent vertices are obeyed.

describe a second, more abstract way to define the Hilbert space.

Let us for convenience suppose the chain is finite, consisting of $N$ anyons. We first consider the space of all trivalent graphs, with endpoints on the $N$ anyons, whose edges are labeled by the nontrivial integer anyon types, and whose vertices satisfy the fusion rules. We take the Hilbert space generated by such graphs (modulo graph isomorphism) and quotient out the subspace generated by the $F$-matrix relations, interpreted as local reconnection rules (see Fig. 3). To relate this graphical picture to the link basis, note that the link basis states can be viewed as labeled trivalent graphs, and that any other labeled trivalent graph can be reduced to a superposition of these using $F$-matrix reconnection rules. The inner product of two graphs is defined by reflecting one of the graphs and concatenating it with the other along the $N$ nodes.

An advantage of this graphical picture of the Hilbert space is that it makes it easy to describe the interaction terms occurring in the Hamiltonian. The Hamiltonian is a sum over $p$ of pairwise interactions between sites $p$ and $p+1$. These are constrained by the $\mathrm{SU}(2)_{k}$ symmetry, and therefore a linear combination of projection operators onto some total topological charge $f$ :

$$
\mathcal{H}=\sum_{p, f} J_{p, p+1}^{f} \mathcal{P}_{p, p+1}^{(f)}
$$

These projection operators $\mathcal{P}$ have a graphical representation, and in the abstract graph basis their action on a particular

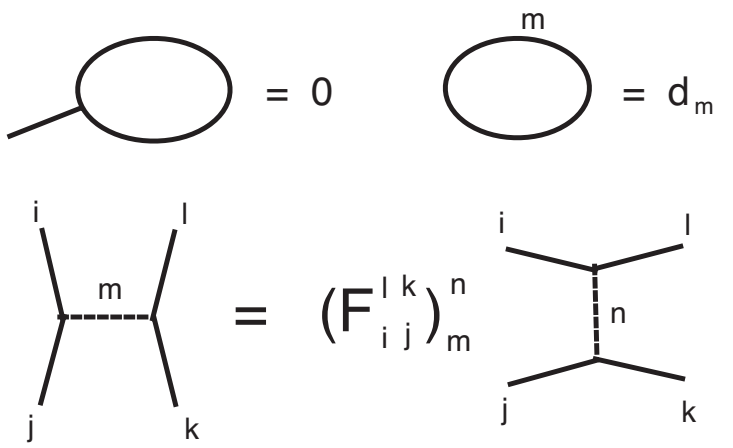

FIG. 3. F-matrix reconnection rules. The first is the so-called "no tadpole" rule, stating that any graph that can be disconnected by the removal of one edge is 0 . The second states that removing a loop with label $m$ is equivalent to multiplying by the quantum dimension $d_{m}=\left(\mathbf{F}_{m m}^{m m}\right)_{0}^{0}$. The third states that by performing the indicated reconnection on any local portion of the graph, we have the stated linear relation among the resulting graphs.

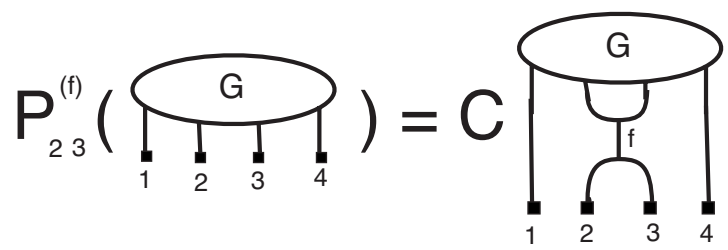

FIG. 4. Graphical representation of a projector onto total topological charge $f$ acting on sites 2 and 3. The oval labeled by $G$ represents a graph as described in the graphical definition of the Hilbert space. The normalization constant $C$ is chosen so as to make $\mathcal{P}$ a projector.

graph $G$ is simply given by concatenation of $\mathcal{P}$ with $G$, up to a normalization constant (see Fig. 4). Their action in the link basis can be worked out by concatenating $\mathcal{P}$ with a particular link graph and then using $F$-matrix rules to reduce the resulting graph to a linear combination of link graphs. In this manner it is apparent that the action of $\mathcal{P}_{p, p+1}$ depends only on $l_{p-1, p}, l_{p, p+1}$, and $l_{p+1, p+2}$; it is thus a three-local operator.

\section{ANALYSIS OF THE DISORDERED SU(2) ${ }_{k}$ CHAIN}

\section{A. Convenient basis for the RG}

We would like to apply the real-space RG procedure to the disordered $\mathrm{SU}(2)_{k}$ chain (15) in hopes of finding infiniterandomness fixed points. Let us first review what happened in our previous analysis ${ }^{12}$ of the case $k=3$, i.e., the Fibonacci chain. $\mathrm{SU}(2)_{3}$ contains one nontrivial anyon of integer charge, the so-called $\tau$ anyon, and the chain is simply an array of these. In the strong randomness limit, we applied the Ma-Dasgupta rule to the strongest bond, which was a projection operator on a pair of neighboring $\tau$ 's. These $\tau$ 's could fuse to one of two possible states, either one with trivial total topological charge or another $\tau(\tau \otimes \tau=0 \oplus \tau)$, and the bond projected onto one of these, leading to either the elimination of both anyons, or their merger into one. In either case, we were left with effectively another realization of the Fibonacci chain, with either one or two fewer sites, allowing us to iterate the procedure.

For $\mathrm{SU}(2)_{k}$ a new complication arises. This time, when we pick the largest bond to decimate, the generic situation is that there are more than two possible fusion products for the corresponding pair of anyons. For example, in $\mathrm{SU}(2)_{5}$, the fusion rules are

$$
\begin{gathered}
1 \otimes 1=0 \oplus 1 \oplus 2, \\
2 \otimes 2=0 \oplus 1, \\
2 \otimes 1=1 \oplus 2 .
\end{gathered}
$$

The first rule, regarding the spin 1 representation, contains three possible fusion products on the right-hand side. We would like to be able to keep just the lowest energy of these, in order to merge the two anyons into a single new effective anyonic site. In general, however, we are not allowed to do this: when there are more than two fusion products, there will be more than one energy splitting associated with the 


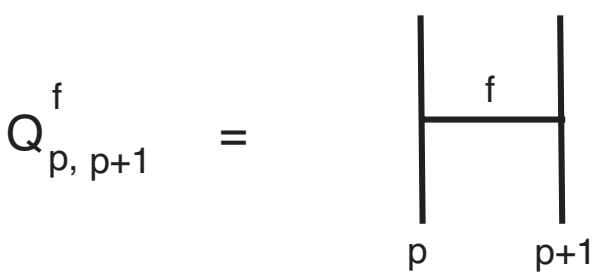

FIG. 5. Graphical definition of the operator $\mathcal{Q}_{p, p+1}^{(f)}$. Note that it is nonzero only when the fusion rules at the $\left(i_{p}, i_{p}, f\right)$ and $\left(i_{p+1}, i_{p+1}, f\right)$ vertices are obeyed.

bond. While we can decimate away the largest one, there may be couplings on other bonds that need to be decimated before the smaller couplings on the original strong bond. This decimation of only one fusion product leads to a situation where on the one hand we need to enforce a constraint on the two anyons, but on the other we are not allowed to merge them into a single effective anyon. This impasse makes it seemingly impossible to carry out an iterative realspace RG analysis. ${ }^{16}$

In the rest of this paper we demonstrate the existence and stability of an infinite-randomness fixed point for $\mathrm{SU}(2)_{k}$ chains that circumvents the above difficulty. The idea is to construct a Hamiltonian out of two-site operators $\mathcal{Q}_{p, p+1}$,

$$
\mathcal{H}=\sum_{p} J_{p, p+1} \mathcal{Q}_{p, p+1}
$$

such that $\mathcal{H}$ retains its form under an RG procedure where we truncate all excited fusion products. In other words, the effective operators generated from first- and second-order decimations are all proportional to $\mathcal{Q}$. The a priori assumption that all such excited states can be truncated, which is not valid in general, is then justified in this particular case if we can show that the resulting RG leads to a strongly disordered set of couplings $J_{p, p+1}$. This is because when we express the operator $\mathcal{Q}_{p, p+1}$ as a linear combination of projection operators

$$
\mathcal{Q}_{p, p+1}=\sum_{f} c_{f} \mathcal{P}_{p, p+1}^{(f)},
$$

the differences between the coefficients $c_{f}$ remain of order 1 , independently of the broadness of the distribution of $\log J_{p, p+1}$. Hence the energy splittings for each bond are all of the same order, and with very high probability all get decimated in one fell swoop in the large disorder limit. The assumption that all excited states can be truncated is then justified and the scheme is self-consistent. Of course, this does not rule out the possibility of more exotic phases where the interactions are not built out of only the $\mathcal{Q}$ operatorsthese phases, if they existed, would not be amenable to treatment by this method.

We now claim that the correct operators to use are the ones defined graphically in Fig. 5 and denoted $\mathcal{Q}^{(f)}$. They can be thought of as an exchange of an anyon of topological charge $f$; their action on any graph $G$ is simply by concatenation with $G$, as for any graphically defined operator. The $\mathcal{Q}^{(f)}$ differ from the projection operators $\mathcal{P}_{p, p+1}^{(g)}$ by an $F$-matrix move, and can of course be expanded as linear
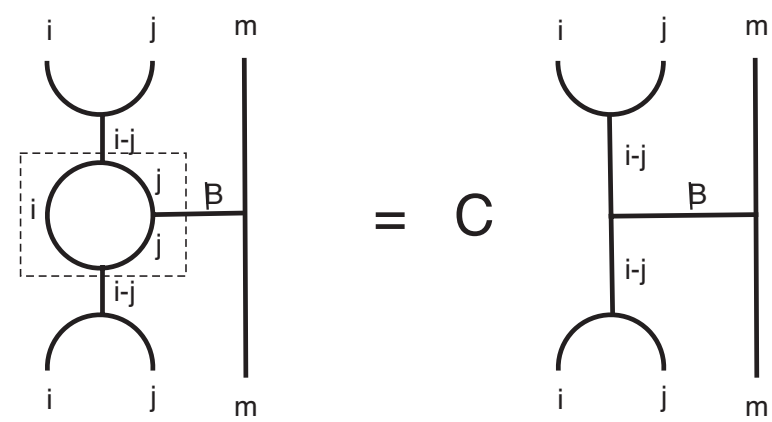

FIG. 6. Graphical representation of the effective operator between the composite $i-j$ and its neighbor $m$, generated at first order in perturbation theory. It is equal to $\mathcal{P}_{12}^{(i-j)} \mathcal{Q}_{23}^{(\beta)} \mathcal{P}_{12}^{(i-j)}$. By performing $F$-matrix manipulations within the dashed box, we see that this effective operator is just a multiple of $\mathcal{Q}_{\text {comp. } 3}^{(\beta)}$.

combinations of the $\mathcal{P}_{p, p+1}^{(g)}$. They all have well-defined scaling under the RG, and, as we show in the Appendix and discuss in more detail below, the most relevant one is $\mathcal{Q}^{(1)}$. Thus from now on we will consider a Hamiltonian of the form

$$
\mathcal{H}=\sum_{p} J_{p, p+1} \mathcal{Q}_{p, p+1}^{(1)},
$$

where the couplings $J_{p, p+1}$ are disordered. In the Appendix, we show

$$
\mathcal{Q}^{(1)}=\sum_{f=|i-j|}^{\min (i+j, k-i-j)} A(f) \mathcal{P}^{(f)},
$$

where

$$
A(f)=\lfloor f\rfloor_{q}^{2}+\lfloor f+1\rfloor_{q}^{2}-\lfloor\mid i-j\rfloor_{q}^{2}-\lfloor i+j+1\rfloor_{q}^{2}
$$

is an increasing function of $f$. Here $i$ and $j$ are the topological charges of the two neighboring anyons on which $\mathcal{Q}^{(1)}$ acts and the $q$ numbers are defined as

$$
\lfloor n\rfloor_{q}=\frac{q^{n}-q^{-n}}{q-q^{-1}},
$$

with $q=e^{\pi i /(k+2)}$.

Thus, depending on the sign of the coupling $J_{p, p+1}$, the anyons at $p$ and $p+1$ can fuse to either an anyon of charge $\left|i_{p}-i_{p+1}\right|$ or one of charge $\min \left(i_{p}+i_{p+1}, k-i_{p}-i_{p+1}\right)$. As discussed above, we decimate all the other fusion products. This scheme will be self-consistent if we show that the system flows to strong disorder, i.e., that the distribution of the $\log J_{p, p+1}$ broadens out. To analyze the flow, we first need to work out the decimation rules.

First, let us focus on first-order decimations. Here the strongest bond $J_{p, p+1}$ fuses the $p$ and $p+1$ anyons into a composite, and the effective interactions between the composite and its neighbors depend on the original $\langle p-1, p\rangle$ and $\langle p+1, p+2\rangle$ interactions. Now, for the Hamiltonian (19) we see from Fig. 6 that these effective interactions are proportional to $\mathcal{Q}_{p-1, p}$ and $\mathcal{Q}_{p+1, p+2}$ - the figure represents graphically the first-order perturbation calculation. ${ }^{12}$ In fact, we can say more: Fig. 6 makes it clear that not only are the $\mathcal{Q}^{(1)}$ 
preserved, but so are all the $\mathcal{Q}^{(\beta)}$. More precisely, if we think of a first-order decimation as a linear mapping from the space of interactions between say $p+1$ and $p+2$ (or $p$ and $p-1)$ to the space of interactions between the composite and $p+2$ (or $p-1$ ), Fig. 6 makes it clear that the operators $\mathcal{Q}_{p, p+1}^{(\beta)}$ are eigenvectors of this mapping.

Second-order decimations follow a similar paradigm, and in the Appendix we show that, again, the $\mathcal{Q}^{(\beta)}$ are eigenvectors of the second-order decimations, and derive the realspace Ma-Dasgupta decimation step, which (for $\mathcal{Q}^{(1)}$ ) reads

$$
\mathcal{H}_{\text {eff }}=C_{j} \frac{J_{1,2} J_{3,4}}{J_{2,3}} \mathcal{Q}_{1,4}^{(1)},
$$

as in Eq. (A18), with $C_{j}$ given by expression (A19). For both first- and second-order decimations, we also calculate in the Appendix the corresponding eigenvalues for all $\beta$, and show that in each case they are maximized for $\beta=1$. Thus $\mathcal{Q}^{(1)}$ is the most relevant operator, which is why we chose to construct the Hamiltonian out of it in Eq. (19). It is stable with respect to perturbations by the $\mathcal{Q}_{p, p+1}^{(\beta)}$ for $\beta \geq 2$, which, having smaller eigenvalues under decimation, are irrelevant.

In fact, the stability argument is not entirely rigorous, but follows the usual line of justification for the validity of strong randomness RG. Basically one can show that the addition of a small amount of $\mathcal{Q}_{p, p+1}^{(\beta)}$ for $\beta>1$ does not change the decimation rules, and so these $\mathcal{Q}_{p, p+1}^{(\beta)}$ decay away under the RG, up to bad spots, or "cancers," that occur with frequency that vanishes with increasing randomness. We can invoke the standard line of reasoning used to justify strong randomness $\mathrm{RG}$ in the first place to argue that these do not destabilize the fixed point, though ultimately this should be decided by numerical simulation.

Thus, with ansatz (19) for the Hamiltonian, we have a consistent framework for the RG that eliminates the potential multitude of widely distributed energy scales associated to each bond. Instead, we have only one energy scale for each bond, multiplying the operator $\mathcal{Q}_{p, p+1}^{(1)}$. In the strong randomness limit, decimation of the strongest bond results, with probability approaching 1 , in the decimation of all its excited states, leaving a 0 or 1 anyons in place of 2 . This decimation process preserves the form of the interactions $\mathcal{Q}_{p, p+1}^{(1)}$. All that we have left to do is to show that under the RG Hamiltonian (19) does indeed flow to strong randomness. This we do in the next subsection.

\section{B. Flow equations}

Let us now solve model (19). To do this, we need to derive the flow equations that describe real-space RG. We are dealing with an ensemble of chains, where not only the coupling strengths and signs, but also the anyon types are chosen according to some probability distribution (see also Ref. 8). We make the ansatz that the coupling strengths, signs, and anyon types are completely uncorrelated from each other, and uncorrelated among the different sites/bonds. Also, for simplicity we analyze only the case in which the bond strength probability distribution is symmetric with respect to sign change. The stability analysis in this framework might in principle miss asymmetric relevant perturbations, and indeed other nonindependent distribution perturbations, but the exact mapping to (a $Z_{k}$ symmetric subspace of) the Damle-Huse model discussed in the next subsection will show that there are none, at least at the level of analysis in Ref. 7.

Let $R(i), i=1, \ldots,(k-1) / 2$ be the probability distribution for the (integer) anyon types, and $P(\beta)$ the logarithmic bondstrength probability distribution, normalized to integrate out to $1 / 2$ because of the two possibilities for the sign of the coupling. Here $\beta=\log (\Omega / J)$ is the logarithmic coupling, and $\Omega$ the energy cutoff. Considering both first- and second-order decimations, we obtain the following infinitesimal transformation for the joint probability distribution $R(i) P(\beta)$ :

$$
\begin{aligned}
R(i) P(\beta) \rightarrow & R(i) P(\beta+d \Gamma)+d \Gamma P(0)(R \otimes R)(i) P(\beta) \\
& +d \Gamma P(0)(R \otimes R)(0) R(i)(P \otimes P)(\beta) .
\end{aligned}
$$

The notation is defined below. Here the first term comes from the cutoff rescaling, the second from first-order decimations, and the third from second-order decimations. Equation (24) is equivalent to the following two norm preserving transformations of $R(i)$ and $P(\beta)$ :

$$
\begin{aligned}
R(i) \rightarrow & R(i)+P(0) d \Gamma(R \otimes R)(i) \\
& -P(0) d \Gamma[1-(R \otimes R)(0)] R(i),
\end{aligned}
$$

$$
\begin{aligned}
P(\beta) \rightarrow & P(\beta)+d \Gamma P^{\prime}(\beta)+P(0) d \Gamma(R \otimes R)(0)(P \otimes P)(\beta) \\
& -P(0) d \Gamma[1-(R \otimes R)(0)] P(\beta) .
\end{aligned}
$$

The notation is as follows. We define the convolution

$$
(P \otimes P)(\beta)=2 \int_{0}^{\beta} d \beta^{\prime} P\left(\beta^{\prime}\right) P\left(\beta-\beta^{\prime}\right)
$$

with an extra factor of 2 to account for normalization of $P$, and we let

$$
(R \otimes R)(i)=\sum_{j, l=1}^{(k-1) / 2} \eta(i, j, l) R(j) R(l),
$$

where $\eta(i, j, l)$ is equal to 1 if $i=|j-l|$ or $i=\min (j+l, k-j$ $-l$ ) and is 0 otherwise (the two possibilities correspond to the two possible signs of the coupling).

We can also write down the integro-differential flow equations corresponding to these infinitesimal transformations (the $\Gamma$ dependence is implicit):

$$
\begin{gathered}
\frac{d R(i)}{d \Gamma}=P(0)(R \otimes R)(i)-P(0)[1-(R \otimes R)(0)] R(i), \\
\frac{d P(\beta)}{d \Gamma}=P^{\prime}(\beta)+2 P(0)(R \otimes R)(0) \int_{0}^{\beta} d \beta^{\prime} P\left(\beta^{\prime}\right) P\left(\beta-\beta^{\prime}\right) \\
-P(0)[1-(R \otimes R)(0)] P(\beta) .
\end{gathered}
$$


A solution to these equations is

$$
\begin{gathered}
R_{0}(i)=\frac{2}{k-1}, \\
P_{0}(\beta)=\frac{k-1}{2 \Gamma} e^{-(k-1) \beta / \Gamma} .
\end{gathered}
$$

Let us analyze the stability of the solution in Eq. (28). First let us look at $R(i)$. Consider a perturbation of the form $R(i)=R_{0}(i)+\epsilon_{i}$. Using the easily derived fact that to linear order $(R \otimes R)(i)=\left(R_{0} \otimes R_{0}\right)(i)-2 \epsilon_{i}$ and the fact that $\Sigma_{i} \epsilon_{i}=0$ we get the RG flow of $\epsilon_{i}$,

$$
\frac{d \epsilon_{i}}{d \Gamma}=-P(0) \epsilon_{i}
$$

which, since $P(0)$ is always positive, shows that $\epsilon_{i}$ always decays. Now consider $P(\beta)$. At linear order the variation in $(R \otimes R)(0)$ vanishes, so the analysis of the stability of $P(\beta)$ is as in all the other examples of strong randomness RG where this solution occurs. ${ }^{5,6}$ As mentioned before, we do not consider perturbations asymmetric with respect to the sign of the coupling, but we expect these to be stable as well by arguments similar to those in Ref. 12 . We also show now via explicit mapping to the Damle-Huse model that they indeed are stable.

\section{RELATION TO THE DAMLE-HUSE DOMAIN WALL MODEL}

As mentioned above, the fixed points we found above must somehow be related to the Damle-Huse fixed points of Abelian spin chains. In this section we present and discuss the mapping between the Damle-Huse domain model with $n=k$ domains and spin $S=(k-1) / 2$, and our $\mathrm{SU}(2)_{k}$ anyonic chains, and show that this mapping gives an equivalence between the permutation symmetric multicritical point of the domain model and the fixed point of the non-Abelian anyonic chain. In addition, we show that the anyonic fixed point is actually a stable phase.

\section{A. Mapping between the two models}

Some intuition for the mapping between the spin- $S$ Damle-Huse chain with $S=(k-1) / 2$ and the $\mathrm{SU}(2)_{k}$ anyon chain can be obtained by inspecting the Bratteli diagram in Fig. 8. Naively, we like to think of the possible topological charges $i=0,1 / 2, \ldots, k / 2$ of the $\mathrm{SU}(2)_{k}$ tensor category as somehow related to spin- $i$ representations of $\mathrm{SU}(2)$. This naive notion is not quite correct, because of the special constraints that the Hilbert space truncation presents. As it turns out, both $i$ and $k / 2-i$ essentially represent the same nontrivial topological charge. Therefore the distinct nontrivial topological charges can be indexed by integer $i$ 's: $i$ $=1,2, \ldots, k-1 / 2$; the half-integer values of $i$ can be turned into integers through $i \rightarrow k / 2-i$. This is, for example, the reason for our ability to restrict our rendition of the $\mathrm{SU}(2)_{5}$ fusion algebra to the rules in Eq. (13) using only the charges 1 and 2 , alongside the trivial (vacuum) charge 0 .
The association of $i=0,1 / 2, \ldots, k / 2$ with spin- $i \mathrm{SU}(2)$ representations provides the correct intuition for the mapping between the anyon model and the spin $(k-1) / 2$ Damle-Huse domain model. In the spin- $(k-1) / 2$ Damle-Huse chain, each site appears as a domain wall between two domains, say $\sigma_{m}$ and $\sigma_{m+1}$-in order to maintain transparent notation we label such a site by the pair $(m, m+1)$. The spin of site $(m, m$ $+1)$ is expected to be

$$
S_{m, m+1}=\frac{\left|\sigma_{m+1}-\sigma_{m}\right|}{2} .
$$

The range of possible spins is $1 / 2 \leq S_{m, m+1} \leq(k-1) / 2$, just like $i$ above, excluding the two trivial (vacuum) charges $i$ $=0, k / 2$. Thus in the mapping to the Damle-Huse model, the natural thing to do is to identify the domain wall spins with the anyon charges. However, we would also like to restrict to the integer topological charges. This is naturally done through the mapping:

$$
f(S)=\left\{\begin{array}{cc}
S & S \in \mathbb{Z} \\
\frac{k}{2}-S & S+\frac{1}{2} \in \mathbb{Z}
\end{array}\right.
$$

with $2 S \in \mathbb{Z}$, an integer, and $0<S<k / 2$. So for example, for $k=5$, we have $f(2)=f(1 / 2)=2$, and $f(1)=f(3 / 2)=1$.

Now let us define the mapping a little more formally. A configuration in the Damle-Huse model is completely specified by a sequence $\left\{\sigma_{m}, \beta_{m}\right\}$ of domains $\sigma_{m} \in 0,1, \ldots, k-1$ and log couplings $\beta_{m}$ between them, since the signs $s(m)$ $= \pm 1$ of the couplings are uniquely determined by this data. As described above, we map this configuration to the sequence $\left\{i_{(m, m+1)}=f\left(\left|\sigma_{m+1}-\sigma_{m}\right| / 2\right)\right\}$ of $\mathrm{SU}(2)_{k}$ anyons, with the $\log$ of the coupling $J_{m}$ between anyons $i_{(m-1, m)}$ and $i_{(m, m+1)}$ given by $\beta_{m}$, and the sign of $J_{m}$ given by

$$
s^{\prime}(m)=(-1)^{\left(\sigma_{m+1}-\sigma_{m-1}\right)} s(m) .
$$

This choice of sign will reproduce the preferred fusion channels upon mapping a spin- $(k-1) / 2$ chain (in its domain wall representation) to an anyonic problem.

This mapping commutes, by construction, with both the first- and second-order real-space RG decimations. Since this claim is the key point in the proof, for clarity, we illustrate it with a specific example in Fig. 7. In the caption we explain how we get the same anyon configuration and couplings, including signs, irrespective of whether we do the decimation in the domain model and then map to the anyon model, or first map to the anyon model and do the decimation there. This means that the two operations commute. Second-order decimations (not illustrated) are even easier to handle. Here, in both the domain model and the anyon model, the sign of the coupling is given by the Ma-Dasgupta rule (3), and hence commute. We have thus defined a mapping from the configuration space of the Damle-Huse model to that of our anyon model, and this mapping respects the RG evolution. It is quite remarkable that despite the two different origins of the interaction couplings' signs (one from the Damle-Huse domain model rules, and one from an $F$-matrix calculation), the signs conspire to make the two operations commute. This is presumably a deep reflection of the fact that the $\mathrm{SU}(2)_{k}$ ten- 


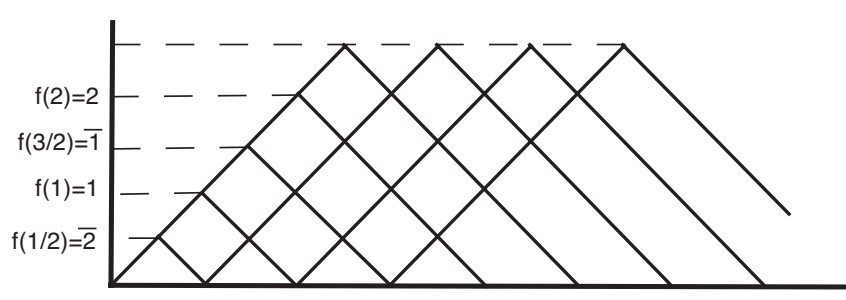

FIG. 7. An example illustrating commutativity of the equivalence mapping between the spin-2 Damle-Huse chain and the $\mathrm{SU}(2)_{5}$ anyon chain and (first order) decimations. A spin-2 configuration has five different domains, while the anyon model has two nontrivial charges: 1 and 2 . The domain model configuration at the upper left has domains labeled by $\sigma=3,1,4,2,1$, signs of couplings $+1,+1,-1$, and strongest bond spanning the 4 domain. These are mapped to the $\mathrm{SU}(2)_{5}$ domain (down arrow), with topological charges following Eq. (31) as $f\left(\frac{\left|\sigma_{m+1}-\sigma_{m}\right|}{2}\right)$, and signs of the couplings following Eq. (32), $s^{\prime}(m)=(-1)^{\left(\sigma_{m+1}-\sigma_{m-1}\right)}$. Decimating bond 4 (right arrow) in the anyon chain follows the principle stated in the Appendix: when two anyons $i$ and $j$ fuse ferromagnetically to $i+j$ $<k / 2$, the effective couplings are the same as the original couplings (as opposed to other cases where the sign may flip). Starting again from the top left corner, carrying out a real-space decimation (right arrow) in the Damle-Huse chain first, and then the mapping to the anyon model (down arrow) results in the same configuration, as illustrated. That this is true in general requires one to check a few more similar cases. We thus see that the mapping from the spin- $(k-1) / 2$ Damle-Huse chain to the $\mathrm{SU}(2)_{k}$ chain and real-space RG steps commute.

sor categories were constructed from the spin representation of SU(2). Also, note that while the Ma-Dasgupta rules in the two models may have different multiplicative constants, leading to a small difference in the logarithmic couplings between the two models, this difference is unimportant in the large disorder limit.

\section{B. Symmetry considerations and elimination of relevant perturbations}

Let us examine the properties of this mapping of configuration spaces. First of all, we claim the map is onto, i.e., given any configuration $\left\{i_{(m-1, m)}, \beta_{m}, s^{\prime}(m)\right\}$, there is a domain configuration $\left\{\sigma_{m}, \beta_{m}, s(m)\right\}$ that maps onto it. To see this, we first pick any $0 \leq \sigma_{1} \leq k-1$. Then we must pick 0 $\leq \sigma_{2} \leq k-1$ such that $f\left(\left|\sigma_{2}-\sigma_{1}\right| / 2\right)=i_{(1,2)}$. It is easy to see that there are precisely two choices of such $\sigma_{2}$ (naively we may think there are four, given the 2 to 1 nature of both $f$ and the absolute value mapping, but two of those choices are not between 0 and $k-1$ ). Now we must similarly choose $\sigma_{3}$; however, in this case we also have to choose it in such a way that the sign $s(2)$ comes out correctly. This constraint uniquely determines $\sigma_{3}$, and in fact all the other $\sigma_{m}$ (for $m$ $>2$ and $m \leq 0$ ) are uniquely determined in this manner.

Thus, we have not only shown that the mapping is onto, but also that each anyon model configuration has precisely $2 k$ domain configurations that map to it, for the $k$ choices of $\sigma_{1}$ above, and the two choices of $\sigma_{2}$. Indeed, this $2 k$-fold degeneracy is easy to understand. First of all, since the anyon charges are functions of only the differences between the $\sigma_{m}$, we can add a constant $c$ to all the $\sigma_{m}$ (mod) $k$ without changing the anyon charges. In fact, this is somewhat subtle. For example, if we have $\sigma_{m}<\sigma_{m+1}$, the corresponding anyon charge is $f\left(\left(\sigma_{m+1}-\sigma_{m}\right) / 2\right)$. Now, if we add a constant $c$ such that $0 \leq \sigma_{m}+c<k$ but $\sigma_{m+1}+c \geq k$, i.e., so that $\sigma_{m+1}$ cycles through, the new anyon type is

$$
\begin{aligned}
f\{ & {\left.\left[\left(\sigma_{m}+c\right)-\left(\sigma_{m+1}+c-k\right)\right] / 2\right\} } \\
& =f\left[k / 2-\left(\sigma_{m+1}-\sigma_{m}\right) / 2\right] \\
& =f\left[\left(\sigma_{m+1}-\sigma_{m}\right) / 2\right]
\end{aligned}
$$

by the definition of $f$. Thus the anyon type still remains invariant. The signs of the domain model couplings also get modified under such a cyclic shift of $\sigma_{m+1}$, but this is precisely canceled by the corresponding modification of the $s^{\prime}(m)=(-1)^{\left(\sigma_{m-1}-\sigma_{m+1}\right)} s(m)$ sign rule. This cycling accounts for a $k$-fold degeneracy; the factor of 2 comes from the flip $\sigma_{m} \rightarrow k-\sigma_{m}$, which preserves absolute values of differences and signs of the couplings as well. Thus the set of $2 k$ preimages of any anyon model configuration is simply an orbit of the dihedral group $\mathbb{D}_{k}$, viewed as a subgroup of the symmetric group $S_{k}$ acting on the configuration space of the Damle-Huse model.

Now that we understand the nature of the mapping in Eqs. (31) and (32), we can explicitly verify that the inverse image of the fixed point ensemble (28) under this mapping is precisely the Damle-Huse fixed point. This confirms that we have a map that identifies the two fixed points and commutes with RG evolution. Furthermore, it means that the physical properties of the two systems are the same, except those that might be affected by the $2 k$ to 1 nature of the mapping. One of these is the existence of relevant perturbations-we will prove that the anyon model has no relevant perturbations, making it a stable phase.

Before giving the formal argument regarding lack of relevant perturbations, we illustrate what happens with a physically appealing picture. Specifically, the domain model has $k-1$ relevant perturbations ${ }^{7}$ each of which can be described in terms of one domain falling out of favor with respect to the rest (there is a linear constraint since they cannot all fall out of favor simultaneously). One might try to construct relevant perturbations of the anyon model by mapping these relevant perturbations of the domain model, as follows: each relevant perturbation can be thought of as a functional on the configuration space of the domain model, so one can, for a given anyon model configuration, sum up the values of the relevant perturbation on all $2 k$ of its pre-images (which are domain model configurations). This sum, however, turns out to be 0 , so no relevant perturbation in the anyon model can be constructed this way.

Let us proceed by providing a formal proof based on symmetry. The proof is by contradiction. Supposing we had a relevant perturbation of the anyon model, we could then pull it back to a relevant perturbation of the domain model [given a map $M: X \rightarrow Y$ of spaces, the pullback map on function spaces $F(Y) \rightarrow F(X)$ is defined by $f \rightarrow g$ where 
$g(x)=f(M(x))]$. Because of the $2 k$ to 1 nature of the mapping, this would yield a $\mathbb{D}_{k}$ symmetric relevant perturbation of the domain model; in particular it would also be $\mathbb{Z}_{k} \subset \mathbb{D}_{k}$ symmetric. The $k-1$ relevant perturbations of the domain model, however, are not $\mathbb{Z}_{k}$ symmetric, because they are described in terms of one of the $k$ domains falling out of favor with respect to the others. Indeed, they form a $k-1$ dimensional nontrivial irreducible representation of $\mathbb{Z}_{k}$. Our putative relevant deformation is $\mathbb{Z}_{k}$ symmetric, i.e., lies in the trivial representation of $\mathbb{Z}_{k}$. Thus we have found a nonexistent relevant perturbation of the domain model, a contradiction. Of course, this analysis does not include possible perturbations by the addition of interactions $\mathcal{Q}^{(f)}$ with $f>1$, but we have already shown that the Hamiltonian is stable with respect to such perturbations in Sec. III, i.e., we showed they are irrelevant. We have therefore realized all of the odd $k S_{k}$ symmetric multicritical points as stable phases in the $\mathrm{SU}(2)_{k}$ anyon chains.

\section{CONCLUSIONS}

In this paper we analyzed in full generality spin chains made of non-Abelian quasiparticles arising in the tensor categories of $\mathrm{SU}(2)_{k}$ for odd $k$, in the limit of strong randomness. We have found a realization of the odd $k S_{k}$ symmetric infinite-randomness multicritical fixed points of Damle and $\mathrm{Huse}^{7}$ as critical stable phases of disordered $\mathrm{SU}(2)_{k}$ anyon chains. We have shown that the $\mathrm{SU}(2)_{k}$ fixed point is stable by analyzing the RG flow equations around it, and also by explicit mapping to the Damle-Huse model. Key in our analysis was our use of a basis of interaction operators $\mathcal{Q}^{(f)}$ that behave well with respect to real-space decimations, i.e., operators $\mathcal{Q}^{(f)}$ that have well-defined scaling under the RG. We found that $\mathcal{Q}^{(1)}$ is the most relevant operator, and thus the only one appearing in the effective Hamiltonian (19) at the fixed point. This effective elimination of all but one interaction operator is what resolves the a priori problem of having a potential multitude of energy scales associated with the multiple fusion products of each neighboring pair of anyons. Indeed, at the fixed point (19) each bond is characterized by only one energy scale: the coefficient in front of the $\mathcal{Q}^{(1)}$ operator.

Recall that the motivation for our study was the search for universality classes of infinite-randomness fixed points. In that sense, our analysis led to a disappointment: the $\mathrm{SU}(2)_{k}$ anyonic chains exhibited the same behavior as random spin- $(k-1) / 2$ chains, as though the differences between the extremely distinct Hilbert spaces of the two systems were essentially washed out in the strong randomness limit, leading to the same infinite-randomness fixed points. Nevertheless, a crucial difference arose: the permutation symmetric fixed points mark stable phases of the $\mathrm{SU}(2)_{k}$ random spin chains, as opposed to unstable points in the ordinary spin chains.

One natural question is then whether $\mathrm{SU}(N)_{k}$ for higher $N$ behaves any differently. It is plausible that using an approach similar to the one in this paper, with the relevant interactions as exchanges of a certain anyon type, will yield the already known fixed points, essentially because the charge of the exchanged anyon will pick out a preferred $\mathrm{SU}(2) \subset \mathrm{SU}(N)$ and will decompose the problem to the $\mathrm{SU}(2)$ cases already studied. This does not, of course, rule out the possibility of more symmetric and exotic fixed points, which may arise with some fine tuning, for instance.

Another line of investigation deals with the relation to the uniform $\mathrm{SU}(2)_{k}$ anyonic chains of. ${ }^{13}$ There, a "topological" symmetry stabilizes an otherwise $k$ multicritical point CFT low-energy spectrum. A physical interpretation of this phenomenon is given in terms of separating out the left and right moving modes of the chain, while creating a different topological liquid between them-the topological symmetry then eliminates relevant tunneling operators between the two modes. One could then add some very weak disorder to this system: at short distances the picture of two modes separated by a topological liquid is preserved, while at long distances the disorder grows and the dynamics is controlled by the infinite-randomness fixed point discussed in this paper. It is interesting to try to find a physical picture for the infiniterandomness phase - perhaps with the intervening liquid having broken up into disconnected islands-which may also yield the stability argument of the fixed points we found.

On the other hand, a picture that is similar to the one of separating the right and left moving modes away from each other but which also applies to the strongly disordered system may provide clues to the understanding of the behavior of non-Abelian anyons interacting on random planar graphs. Indeed, it would be interesting to see if any of the ideas developed in this paper have application to, say, a two (or higher) dimensional disordered lattice of anyons. So far we have not made progress in this direction.

\section{ACKNOWLEDGMENTS}

We would like to thank John Preskill and Simon Trebst for useful discussions. Also, we would especially like to thank David Huse for useful discussions during the early part of this work. H.-H.L. and P.T. were supported by the Summer Undergraduate Research Foundation at the California Institute of Technology. L.F. and G.R. would like to acknowledge support from the Institute for Quantum Information under NSF Grants No. PHY-0456720 and No. PHY0803371, and from the Packard Foundation.

\section{APPENDIX}

In this appendix, we derive Eq. (20) for the energy spacing between the different fusion channels in the $\mathcal{Q}^{(1)}$ interaction, show that $\mathcal{Q}^{(1)}$ is the most relevant operator for both first- and second-order decimations, and derive the sign rules for the changes of the sign of the couplings under first-order decimations. For the analysis we will need the following expression $^{17}$ for the $F$ matrix (or $6-j$ symbols) of $\mathrm{SU}(2)_{k}$ : 


$$
\begin{aligned}
\left(F_{j_{1} j_{2} j_{j_{12}}^{j j_{3}} j_{23}=}\right. & \sqrt{\left.\left\lfloor 2 j_{12}+1\right\rfloor_{q} L 2 j_{23}+1\right\rfloor_{q}} \Delta\left(j_{1}, j_{2}, j_{12}\right) \Delta\left(j_{12}, j_{3}, j\right) \Delta\left(j_{2}, j_{3}, j_{23}\right) \Delta\left(j_{1}, j_{23}, j\right) \\
& \times \sum_{z}\left\{\frac{(-1)^{z}\lfloor z+1\rfloor_{q} !}{\left\lfloor z-j_{1}-j_{2}-j_{12}\right\rfloor_{q} !\left\lfloor z-j_{12}-j_{3}-j\right\rfloor_{q} !\left\lfloor z-j_{2}-j_{3}-j_{23}\right\rfloor_{q} !\left\lfloor z-j_{1}-j_{23}-j\right\rfloor_{q} !}\right. \\
& \left.\times \frac{1}{\left\lfloor j_{1}+j_{2}+j_{3}+j-z\right\rfloor_{q} !\left\lfloor j_{1}+j_{12}+j_{3}+j_{23}-z\right\rfloor_{q} !\left\lfloor j_{2}+j_{12}+j+j_{23}-z\right\rfloor_{q} !}\right\}
\end{aligned}
$$

Here the $q$ numbers are defined as in Eq. (22). The sum is over all $z$ for which all the $q$ factorials are well defined, i.e., such that the arguments are $\geq 0$, and

$$
\Delta(a, b, c):=\sqrt{\frac{\lfloor-a+b+c\rfloor_{q} !\lfloor a-b+c\rfloor_{q} !\lfloor a+b-c\rfloor_{q} !}{\lfloor a+b+c+1\rfloor_{q} !}} .
$$

Let us first analyze the operator $\mathcal{Q}_{p, p+1}^{(1)}$. To simplify notation, we denote $\left\{i_{p}, i_{p+1}\right\}$ by $\{i, j\}$ and drop the subscripts on $\mathcal{Q}^{(\beta)}$ and the projectors $\mathcal{P}^{(\beta)}$. Applying an $F$-matrix move and noting the normalization on the projector $\mathcal{P}_{p, p+1}^{(f)}$, we obtain

$$
\mathcal{Q}^{(1)}=\sum_{f=|i-j|}^{\min (i+j, k-i-j)} \frac{\left(F_{i i}^{j j}\right)_{1}^{f}}{\left(F_{i i}^{j j}\right)_{0}^{f}} \mathcal{P}^{(f)} .
$$

We would like to know the $f$ dependence of the coefficient in front of $\mathcal{P}^{(f)}$. Plugging into Eq. (A1), up to an $f$-independent prefactor, the coefficient is

$$
\left(\lfloor f\rfloor_{q}^{2}+\lfloor f+1\rfloor_{q}^{2}-\lfloor\mid i-j\rfloor_{q}^{2}-\lfloor i+j+1\rfloor_{q}^{2}\right)
$$

giving us Eq. (20) as desired. Note that the quantity in the brackets is an increasing function of $f$.

Let us now work out the decimation rules for Hamiltonian (19), and in particular show that the $\mathcal{Q}$ operators are eigenvectors with respect to decimations, with $\mathcal{Q}^{(1)}$ having the largest eigenvalue. Pick the largest coupling $J_{p, p+1}$. According to Eq. (A4), anyons $p$ and $p+1$ will be fused into an anyon of total topological charge $\left|i_{p}-i_{p+1}\right|$ for $J_{p, p+1}>0$ and into an anyon of charge $\max \left(i_{p}+i_{p+1}, k-i_{p}-i_{p+1}\right)$ for $J_{p, p+1}$ $<0$. Suppose first that this composite anyon charge is nonzero. In this case we need to use first-order perturbation theory to work out the effective coupling of this composite anyon to its neighbors. For simplicity we only consider $i_{p+2}$, the neighbor to the right, and to simplify notation we denote $i_{p}, i_{p+1}, i_{p+2}$ by $i, j, m$.

Let us first deal with a specific case, say, having $i>j$ fuse to $i-j$. According to first-order perturbation theory, the effective coupling between $i-j$ and $m$ is given by

$$
\mathcal{P}_{p, p+1} \mathcal{Q}_{p+1, p+2}^{(1)} \mathcal{P}_{p, p+1} .
$$

Graphically we can see (Fig. 6) that this effective coupling is still a multiple of $\mathcal{Q}^{(1)}$. There is a finite factor in front of the coefficient, as well as a possible sign, but we already see that form (19) is preserved by first-order decimations. In fact, it will be useful to go a little further and examine the effect of such a decimation on the other interactions $\mathcal{Q}_{p+1, p+2}^{(\beta)}$. Again, we graphically see that the effective coupling will be a con- stant multiple of $\mathcal{Q}_{p+1, p+2}^{(\beta)}$. We are only interested in the sign and $\beta$ dependence of this constant, which turns out to be equal to the constant $C$ in Fig. 6, this being the evaluation of the graph in the dashed box. This is

$$
\mu(\beta)=\frac{\left(F_{\alpha j}^{\alpha j}\right)_{i}^{\beta}}{\left(F_{j j}^{j j}\right)_{0}^{\beta}}
$$

where $\alpha$ is the fusion product of $i$ and $j$, in this case $\alpha=i$ $-j$. Plugging into Eq. (A1), we see that the $\beta$ dependence of Eq. (A6) is

$$
\begin{array}{r}
\mu(\beta)=(-1)^{\beta}\left(\lfloor 2 j-\beta\rfloor_{q} !\lfloor 2 i-2 j+\beta+1\rfloor_{q}\right. \\
\left.!\lfloor 2 j+\beta+1\rfloor_{q} !\lfloor 2 i-2 j-\beta\rfloor_{q} !\right)^{-1 / 2} .
\end{array}
$$

We observe that the magnitude of this quantity decreases as a function of $\beta$. To see this, note that

$$
\begin{aligned}
\mu(\beta) / \mu(\beta-1) & =-\left(\frac{\lfloor 2 j-\beta+1\rfloor_{q}}{\lfloor 2 j+\beta+1\rfloor_{q}}\right)^{1 / 2}\left(\frac{\lfloor 2 i-2 j-\beta+1\rfloor_{q}}{\lfloor 2 i-2 j+\beta+1\rfloor_{q}}\right)^{1 / 2} \\
& =-h(j) h(i-j) .
\end{aligned}
$$

It is easy to see using the explicit expression for the $q$ numbers in terms of roots of unity that $h(j)=h(k / 2-j)^{-1}$ and that $h(j)$ is an increasing function of $j$. Therefore

$$
h(j) h(i-j) \leq h(j) h(k / 2-j)=1 .
$$

Thus we have shown that $\mathcal{Q}^{(1)}$ has the highest eigenvalue under first-order decimations. We have only considered the case $i>j, \alpha=i-j$, but similar arguments apply to other cases (for the case of $\alpha=k-i-j$ we need to use the symmetries of the $F$ matrix discussed in Ref. 18) to show that $\mu(\beta)$ decreases as a function of $\beta$.

The one thing we need to know explicitly for the mapping to the Damle-Huse model is whether first-order decimations flip the sign of the neighboring couplings, when those are of the form $\mathcal{Q}^{(1)}$. For the case just considered, $i>j, \alpha=i-j$, the sign is $(-1)^{\beta}=-1$. For the rest of the cases the sign can be read off from the factors of $(-1)^{z}$ in Eq. (A1): when $i$ $<j, \alpha=j-i$, the sign is +1 ; when $\alpha=i+j<k / 2$, the sign is +1 ; and when $\alpha=k-i-j<k / 2$ the sign is -1 .

Consider now the second-order decimations. The setup here is that we have four consecutive anyons, with the middle two antiferromagnetically fusing to the trivial channel, so we can label their topological charges $a, j, j, b$. The picture is as in Fig. 9. The bare Hamiltonian is 


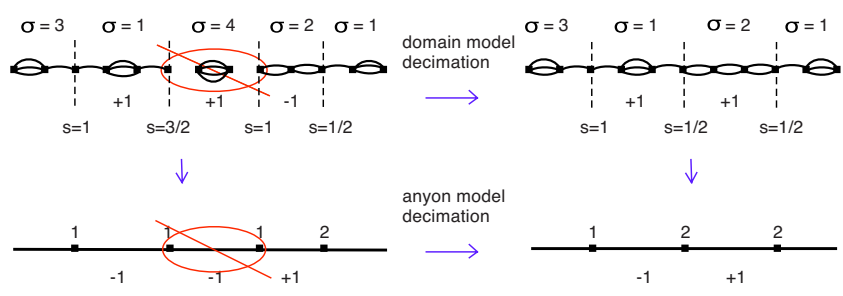

FIG. 8. (Color online) Bratelli diagram for $\mathrm{SU}(2)_{5}$. The mapping $f$ defined below simply reflects the topological charges along the vertical axis for noninteger arguments, mapping noninteger charges to integers. Bars denote the cases where the argument was noninteger and hence the action of $f$ nontrivial.

$$
H_{0}=J_{2}\left(\mathcal{Q}_{2}^{(1)}-\left\langle\mathcal{Q}_{2}^{(1)}\right\rangle\right),
$$

where $\left\langle\mathcal{Q}_{2}^{(1)}\right\rangle$ is the expectation value of $\mathcal{Q}_{2}^{(1)}$ in the state where the fusion product of the two $j$ 's is trivial. The interaction Hamiltonian is

$$
H^{\prime}=J_{1} \mathcal{Q}_{1}^{\beta}+J_{3} \mathcal{Q}_{3}^{\beta} \text {. }
$$

Note that the two interactions must have the same $\beta$ by charge conservation (see Fig. 10). The induced effective Hamiltonian between $a$ and $b$ at second order is

$$
H_{\mathrm{eff}}=\mathcal{P} H^{\prime} H_{0}^{-1} H^{\prime} .
$$

$H_{\text {eff }}$ acts on the subspace where the two middle anyons, both of charge $j$, fuse to the trivial channel. The inverse $H_{0}^{-1}$ is well defined in this expression because $H^{\prime}$ acting on this subspace gives a vector orthogonal to the subspace. We have, up to a multiple of the identity operator,

$$
\begin{aligned}
H_{\mathrm{eff}}= & \frac{J_{1} J_{3}}{J_{2}} \mathcal{P} \mathcal{Q}_{1}^{\beta}\left(\mathcal{Q}_{2}^{(1)}-\left\langle\mathcal{Q}_{2}^{(1)}\right\rangle\right)^{-1} \mathcal{Q}_{3}^{\beta}+\frac{J_{1} J_{3}}{J_{2}} \mathcal{P} \mathcal{Q}_{3}^{\beta}\left(\mathcal{Q}_{2}^{(1)}\right. \\
& \left.-\left\langle\mathcal{Q}_{2}^{(1)}\right\rangle\right)^{-1} \mathcal{Q}_{1}^{\beta} .
\end{aligned}
$$

Using the $F$-matrix we calculate that

$$
\left(\mathcal{Q}_{2}^{(1)}-\left\langle\mathcal{Q}_{2}^{(1)}\right\rangle\right)^{-1}=\sum_{\gamma}\left(\frac{\left(F_{j j}^{j j}\right)_{1}^{\gamma}}{\left(F_{j j}^{j j}\right)_{0}^{\gamma}}-\frac{\left(F_{j j}^{j j}\right)_{0}^{1}}{\left(F_{j j}^{j j}\right)_{0}^{0}}\right)^{-1} \mathcal{P}^{(\gamma)}
$$

From Fig. 9 we then see that the effective operator between $a$ and $b$ is

$$
\begin{aligned}
& \text { - } J_{1} Q_{1}^{\beta} \text { - } J_{2} Q_{2}^{1} \text { - } J_{3} Q_{3}^{\beta} \\
& \text { a } \\
& \text { j } \\
& \text { j } \\
& \text { b }
\end{aligned}
$$

FIG. 9. The setting for second-order decimations. The antiferromagnetic coupling $J_{2}$ is much larger than $J_{1}$ and $J_{3}$, causing the two middle anyons to combine into a charge singlet.

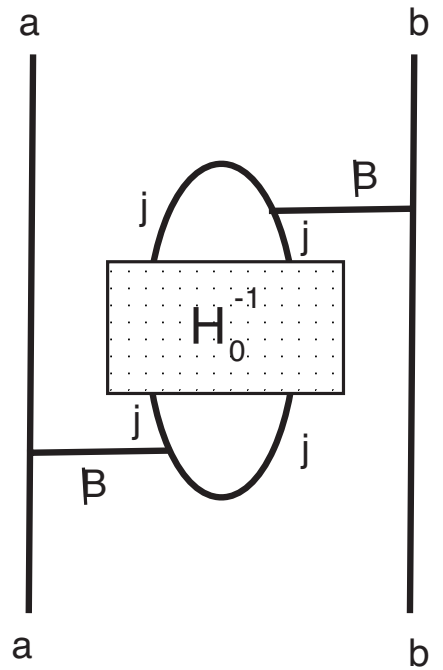

FIG. 10. Graphical expression for the effective interaction between $a$ and $b$ generated at second order in perturbation theory. Here $H_{0}$ is as defined in the text. Its inverse is computed and expressed in graphical form by expanding in projection operators. This is one of the two terms that contribute to an energy splitting between the fusion products of $a$ and $b$. Note that both the original interaction with $a$ and $b$ exchange the same topological charge $\beta$-otherwise the second-order correction is zero by topological charge conservation, as is clear from the figure.

$$
c\left(\frac{\left(F_{j j}^{j j}\right)_{1}^{\beta}}{\left(F_{j j}^{j j}\right)_{0}^{\beta}}-\frac{\left(F_{j j}^{j j}\right)_{0}^{1}}{\left(F_{j j}^{j j}\right)_{0}^{0}}\right)^{-1} \mathcal{Q}^{(\beta)},
$$

where $c$ is a constant containing the normalization of the projection operator $\mathcal{P}^{(\beta)}$ relative to its graphical representation, times the numerical factor one gets from reducing the portion of the graph in Fig. 10 between the two $\beta$ 's using $F$-matrix moves. The product in front of $\mathcal{Q}^{(\beta)}$ comes out to

$$
\left(\left(F_{j j}^{j j}\right)_{1}^{\beta}-\frac{\left(F_{j j}^{j j}\right)_{0}^{1}}{\left(F_{j j}^{j j}\right)_{0}^{0}}\left(F_{j j}^{j j}\right)_{0}^{\beta}\right)^{-1} \text {. }
$$

Evaluating this expression using Eq. (A1) we see that, up to $\beta$-independent factors, it is equal to

$$
(-1)^{\beta}\lfloor 2 \beta+1\rfloor_{q}^{-1 / 2}\left(\lfloor\beta\rfloor_{q}^{2}+\lfloor\beta+1\rfloor_{q}^{2}-1\right)^{-1} .
$$

Though this is not a decreasing function of $\beta$, both of the factors are minimized in absolute value at $\beta=1$, so the absolute value of the expression is maximized at $\beta=1$. Thus we have shown that second-order decimations also have the $\mathcal{Q}^{(\beta)}$ as eigenvectors, and $\mathcal{Q}^{(1)}$ has the highest magnitude eigenvalue. The second-order decimation rule for $\beta=1$ is then

$$
H_{\mathrm{eff}}=\frac{J_{1} J_{3}}{J_{2}} C_{j} \mathcal{Q}_{1,4}^{(1)},
$$

where

$$
C_{j}=\frac{\lfloor 2\rfloor_{q}^{2}}{\lfloor 3\rfloor_{q}}\left(\lfloor 2 j+1\rfloor_{q}-\lfloor 2 j+1\rfloor_{q}^{-1}\right)^{-1} .
$$


${ }^{1}$ A. Yu. Kitaev, Ann. Phys. 303, 2 (2003).

${ }^{2}$ G. P. Collins, Sci. Am. 4, 57 (2006).

${ }^{3}$ A. Feiguin, S. Trebst, A. W. W. Ludwig, M. Troyer, A. Kitaev, Z. Wang, and M. H. Freedman, Phys. Rev. Lett. 98, 160409 (2007).

${ }^{4}$ S. Trebst, E. Ardonne, A. Feiguin, D. A. Huse, A. W. W. Ludwig, and M. Troyer, Phys. Rev. Lett. 101, 050401 (2008).

${ }^{5}$ D. S. Fisher, Phys. Rev. B 50, 3799 (1994).

${ }^{6}$ D. S. Fisher, Phys. Rev. B 51, 6411 (1995).

${ }^{7}$ K. Damle and D. A. Huse, Phys. Rev. Lett. 89, 277203 (2002).

${ }^{8}$ C. Monthus, O. Golinelli, and T. Jolicoeur, Phys. Rev. B 58, 805 (1998).

${ }^{9}$ R. A. Hyman and K. Yang, Phys. Rev. Lett. 78, 1783 (1997).

${ }^{10}$ G. Refael, S. Kehrein, and D. S. Fisher, Phys. Rev. B 66, 060402(R) (2002).

${ }^{11}$ N. E. Bonesteel and K. Yang, Phys. Rev. Lett. 99, 140405 (2007).
${ }^{12}$ L. Fidkowski, G. Refael, N. Bonesteel, and J. Moore, Phys. Rev. B 78, 224204 (2008).).

${ }^{13}$ C. Gils, E. Ardonne, S. Trebst, A. W. W. Ludwig, M. Troyer, and Z. Wang, arXiv:0810.2277 (unpublished).

${ }^{14}$ S. K. Ma, C. Dasgupta, and C. K. Hu, Phys. Rev. Lett. 43, 1434 (1979).

${ }^{15}$ C. Dasgupta and S. K. Ma, Phys. Rev. B 22, 1305 (1980).

${ }^{16}$ This can be circumvented, however, by renormalizing the topological charges such that a certain fusion channel is eliminated. For instance, if we want to exclude the $1 \otimes 1 \rightarrow 2$ channel between two sites, we can demote the two sites from charge 1 to 2 , with the fusion channels now possible $2 \otimes 2=0 \oplus 1$ (this follows the procedure first suggested in Ref. 8). In the limit of strong disorder, however, this is not necessary.

${ }^{17}$ J. K. Slingerland and F. A. Bais, Nucl. Phys. B 612, 229 (2001).

${ }^{18}$ J. K. Slingerland, http://www.stp.dias.ie/slingerland/thesis.pdf 